\title{
Environmentally benign chemistry and chemical laboratory construction in Colleges and Universities
}

\author{
Qinghua Li \\ Nanchang institute of technology \\ Nanchang city, Jiangxi province 330108
}

Key words: environmentally benign chemistry, pollution, laboratory, construction

\begin{abstract}
The environmental problem has become one of the serious challenges which human faced, chemical laboratory in colleges and universities is a serious chemical pollution. Therefore, construction of environmentally benign type laboratory is the only way to realize sustainable development of laboratories, has great significance for improving the environment. Schools should vigorously construct environmentally benign type laboratories, truly reflect the use value and social function of laboratory, in the service of human learning and research activities.

Environmentally Benign Chemical also is known as Green Chemistry, Environmentally Friendly Chemistry or Clean Chemistry, uses chemical technology and methods to reduce or eliminate the use and production of chemical raw materials, catalysts, solvents and reagents that are harmful to human health and ecological environment. The ideal of Environmentally Benign Chemical is no longer to use toxic and harmful substances, no longer to produce waste, no longer to deal with waste, it's a chemistry to prevent pollution from the source. Chemical laboratory is an important place to carry out the teaching and scientific research, be engaged in research and development, is the important base to cultivate chemical talents. But at present, the waste produced by various kinds of chemical laboratories in Colleges and universities in our country is discharged into the environment directly, environmental problem of chemical laboratory has become the focus of widespread attention. Construction of environmentally benign type laboratory is only way to realize sustainable development of laboratories.
\end{abstract}

\section{Set waste gas detection device, not up to standard can't be discharged}

The emission of waste gas in chemical laboratory is more, generally, the emission of toxic gases in laboratory are directly discharged into the atmosphere through the fume cupboard, like toxic gases such as sulfur dioxide, hydrogen sulfide, hydrogen chloride and so on, which directly cause the pollution of the atmosphere. The setting of fume cupboard should have the function of waste gas treatment, toxic and harmful gases must be treated before discharge, through purification absorption device. For the harmful gases, such as sulfur dioxide, hydrogen sulfide, chlorine, hydrogen chloride, hydrogen fluoride and so on can be absorbed by lime water. To purchase toxic and harmful gas detection device, the concentration of toxic gases is qualified, then can be discharged, to prevent pollute the environmental.

\section{Waste liquid discharge setting separate discharge port, detection on a regular basis}

The use of chemicals in laboratory is large, and chemicals have various types, the water washed the instruments contains a lot of chemicals, mixed waste water discharge to the sewer, will 
inevitably produce a variety of pollution hazard. Therefore, should set up a separate discharge port, discharge into a fixed sewage pool, detect the waste water on a regular basis, after achieved the standard, then can be discharged into the environment. The waste liquid in laboratory should be implemented the classification recovery and storage, through the treatment of neutralization, precipitation, oxidation and so on, adopts biological degradation, oxidation, activated sludge process to deal with organic waste liquid, to realize no pollution discharge of waste liquid.

\section{Reasonable storage of toxic substances, collection on a regular basis}

The storage of chemicals used in this laboratory with highly toxic chemicals and radioactive substances must have a dedicated facilities and dedicated cabinets, and managed by specially-assigned staff. According to the type and nature of goods to set corresponding measures of the ventilation, explosion-proof and temperature control. Chemicals storage quantity should have strict rules, the collection and use of items should have strictly registered, the quantity of highly toxic chemicals used one time should be fully reflected in the experiment, the rest can't be discarded at will, should return to specially-assigned staff to manage in a time. The warehouse of storing chemicals should take ventilation measures, reduce the concentration of toxic substances in the air, but should not simply discharge the high concentration polluted gas into the outdoor directly, should set corresponding purification device. The storage time of chemical should not be too long, the decomposed and degenerative chemicals should be timely recovery processed, to avoid a large number of hoarding. Residual chemicals in the process of experiment and the vessel stored the medicines should be timely recovery processed. Toxic waste residue should be buried in fixed location of underground, should not be taken as ordinary garbage to discard at will. For radioactive material, should have dedicated radiation protection devices to save, avoid radioactive sources to be directly exposed in the environment.

\section{Environmental protection of experimental equipment, effectively control the pollution sources}

When purchase the laboratory equipment, should take the environmental factors into consideration. For example, using closed distillation unit, to reduce the leaks of harmful gas. Use the instrumental analysis, put chemical reagents sealed in instrument, to reduce the contact with the environment. Such as carbon sulfur analyzer, the samples under the high temperature through oxygen to oxidize in furnace, make the carbon and sulfur within these samples oxidized to sulfur dioxide, carbon dioxide and carbon monoxide, the generated oxide through the dust and water purification device into the sulfur detection pool by oxygen to detect the sulfur, since then, the mixed gases are into a heating catalyst furnace together, through catalytic reduction transformation, after mixed gases entered into the sulfur removal test tube, guide into carbon detection pool to detect carbon, the residual gases through analyzer discharged to the outside. The system is closed in the whole experimental process, reduced the leak of the gases, poisonous and harmful gases can be concentrated to discharge, reduced the pollution of indoor and outdoor environment.

\section{Equipped with full-time environmental testing personnel, strengthen the management of laboratory environment}

Laboratory management should not focus on prevention, and should focus on fundamental measures___ governance, should take effective governance measures, minimize pollution level of laboratory, can't discharge the poisonous and harmful substances into the environment at will. Some protective measures should not be confined to indoor, should set a full-time professional 
environmental protection personnel to carry out daily monitoring of environmental protection in the laboratory, to achieve institutionalization of environmental protection measures and the standardization of environmental management, bring environmental targets into the laboratory qualification certification system. Follow the internationally accepted standards of 《chemical testing standards》, 《specifications and principles of qualified laboratory (GLP)》 and so on, build perfect support system of laboratory environment management technology. For a long time, lack of perfect prevention regulations for laboratory environment protection problems, monitoring measures is not enough for the environmental protection department, lead to laboratory environmental problems to become a blind spot. Schools should vigorously construct the environmental protection laboratory, equip with professional environmental protection talents, achieve standard discharge for the waste in the laboratory, establish and improve the various environmental protection measures in the laboratory, protect human survival environment, only can truly reflect the use value and social function of laboratory, in the service of human learning and research activities.

\section{Construction of environmentally benign laboratory}

\section{Construct environmental benign laboratory}

When colleges and universities build, rebuild and expand the chemistry laboratory, must take the design and construction of "three wastes" treatment facilities into consideration while building main engineering of the laboratory, the two should be put into use at the same time, it can effectively control the generation of new pollution source. The construction of "three wastes" treatment facilities is the premise of environmentally benign laboratory, also is an important indicator of chemistry laboratory evaluation in colleges and universities. While constructing the "three wastes" treatment facilities, to formulate relevant systems of the "three wastes" treatment, clear their duties, distribute the duties to people and carry out regular inspections, only in this way can ensure the construction of environment benign chemistry laboratory from the view of hardware.

Construct environmental benign chemistry experiment teaching materials

Existing chemistry experiment teaching materials are very little in the new field of environmental benign chemistry, most of the teaching materials do not reflect green requirements, therefore, the tasks of chemistry experiment teaching materials content updates and teaching material knowledge system construction is urgently needed. Strengthening the construction and reform of environmentally benign chemistry experiment teaching materials, makes the teaching materials content reflected the course content, system reform and the results of experimental teaching reform and research results, not only reflect basic characteristics, but also have advanced nature, not only embody the updates of discipline connotation and experiment content, but also reflect the experimental techniques and methods of new technology, new methods and new equipment. Experimental teaching materials should fully embody the characteristics of environment benign chemistry within the principles of quality assurance. According to the requirements of the chemistry experiment teaching outline, give full consideration to the toxicity of reagents and products, consider the pollution "three wastes" produced in the whole process to the environment, as far as possible to choose experimental projects with low toxicity, less polluting and post-processing easy. Vigorously promote the micro experiment, not only can greatly reduce the experiment cost, but also can improve the teaching quality, reduce the waste amount, reduce the pollution of the environment, shorten the test time, be safe and reliable. Reasonably arrange the experimental sequence, make the product of previous experiment as the raw materials of next experiment, at the same time pay attention to the 5R principles of green chemistry experimental 
drugs used, namely reduce, reuse, rejection, recycling and regeneration, reduce the pollution to the environment.

Modern teaching means assist chemistry experiment teaching

Environmental benign chemistry laboratory should use modern technology and advanced experiment teaching means, make full use of the network, use computer aided teaching experimental software and multimedia teaching course ware, expand and use experiment technology means with virtual and simulation, at the same time for the necessary experiment to combine the virtual and simulation experiment with actual experiment. The chemistry experiment teachers can penetrate into the concept of green chemistry in the process of teaching experiment, use multimedia course ware or video to explain what pollution must be payed attention in the experiments, and how to deal with and harm. For the experiment with great pollution let students simulate in computer. For the comprehensive experiment, let the students familiar with the experimental process by computer, then process experiment again, improve the success rate of experiments, reduce the waste of reagent, reduce the experiment accident rate, prevent the happening of the pollution.

Modern instruments and equipment used in the chemistry experiment process

Modern instruments and equipment used in the chemistry experiment, has opened up a new research field for realizing the green chemistry. Research and develop series chemistry experimental instruments and equipment with the function of environmental protection, design and produce complete set of experimental apparatus with full enclosed, prevent pollution to damage the environment, and can timely eliminate pollution, this is a historical task put forward to chemistry workers by the green chemistry. Using instruments and equipment which can improve the reaction rate, such as microwave, ultrasonic and so on, shorten the reaction time, reduce pollution, have the important development prospects. Using digital beckmann thermometer and digital pressure gauge, change the traditional way which use mercury thermometer to measure temperature, use beckman thermometer to measure temperature difference and use mercury pressure to measure pressure or differential pressure, establish a mercury-free laboratory, eliminate pollution that instruments may cause.

\section{Conclusion}

Green chemistry experiment is the inevitable trend of the development of the chemistry experiment teaching, is the important content of green chemistry research, is the best choice of reducing laboratory pollution and protecting the environment. But at the same time, should clearly recognize that it is a system engineering, is a long-term and arduous task. The development of green chemistry is an inexorable historical trend in the chemistry history, chemistry laboratory in colleges and universities should take green chemistry as development direction to accelerate the construction.

\section{References}

[1]Anastas P.T. and Williamson T.C. Green chemistry-designing chemistry for the environment, ACS Symposium Series 626 ${ }^{\text {th }}$. Washington DC. American Chemical Society, 1996.

[2]Rong Yang, Xuanjun Wang. Several ideas on the construction of green chemistry laboratory. Laboratory research and exploration, 2003. 
[3]Wentian Sun, Zhonglei Xie, Xiaobo Zhao. Adsorption properties of excess sludge and its application in waste water treatment [J]. Environmental protection, 2008 (8).

[4]Guomei Zhou, Zhipeng Tang. Research on the mechanism and policy of environment optimizing economic development [J]. Environmental protection, 2008 (10).

[5]Bing Zhu, Tianlong Wu. The new direction of chemistry experiment reform - micro-scale experiment. Chemistry, 1991, (9).

[6] Lin Bai. Green chemistry experiment. Chemistry education, 2002, 23 (7).

[7]Rong Yang, Xuanjun Wang. Several ideas on the construction of green chemistry laboratory. Laboratory research and exploration, 2003, 22 (3). 